\title{
Screened Poisson Equation for Image Contrast Enhancement
}

\author{
Jean-Michel Morel ${ }^{1}$, Ana Belén Petro ${ }^{2}$, Catalina Sbert ${ }^{3}$ \\ ${ }^{1}$ CMLA, ENS Cachan (France) (morel@cmla.ens-cachan.fr) \\ ${ }^{2}$ University of Balearic Islands (Spain) (anabelen.petro@uib.es) \\ ${ }^{3}$ University of Balearic Islands (Spain) (catalina. sbert@uib.es) \\ Communicated by Yohann Tendero Demo edited by Jose-Luis Lisani
}

\begin{abstract}
In this work we propose a discussion and detailed implementation of a very simple gradient domain method that tries to eliminate the effect of nonuniform illumination and at the same time preserves the images details. This model, which to the best of our knowledge has not been explored in spite of its simplicity, acts as a high pass filter. We show that with a single contrast parameter (which keeps the same value in most experiments), the model delivers state of the art results. They compare favorably to results obtained with more complex algorithms. Our algorithm is designed for all kinds of images, but with the special specification of making minimal image detail alteration thanks to a first order fidelity term, instead of the usual zero order term. Experiments on non-uniform medical images and on hazy images illustrate significant perception gain.
\end{abstract}

\section{Source Code}

The source code and the online demo are accessible at the IPOL web page of this article ${ }^{1}$. In this link an ANSI C source code is available for download which produces the same results as the demo. Compilation and usage instructions are included in a README.txt file.

The online demo allows one to try Screened Poisson Equation on any uploaded image choosing the tradeoff parameter $\lambda$ and the level of saturation of the simplest color balance $s$. By default the values of $\lambda$ and $s$ are 0.0001 and 0.1 respectively.

Keywords: color enhancement; Poisson equation; gradient-based methods; non-uniform illumination; color perception theory

\footnotetext{
${ }^{1}$ https://doi.org/10.5201/ipol.2014.84
} 


\section{Introduction}

Modifying image gradients before reconstructing the image is the key idea of the Retinex theory of Land and McCann [10] which argues that the human visual system is sensitive to illumination differences and not to absolute luminance. Since the ground breaking reconstruction algorithm proposed as a "Poisson image editing" [16], many applications use this technique, for high dynamic range compression [3], Retinex [14], [12], contrast enhancement [15], [1], shadow removal [4], etc.

The basic idea of the Poisson editing reconstruction is to find a function $u$ whose gradient field best approximates a given vector field $\mathbf{V}$ in the $L_{2}$ norm. This problem can be solved by minimizing the functional

$$
\int_{\Omega}\|\nabla u-\mathbf{V}\|^{2} d x
$$

leading to a Poisson equation. Strong illumination variations affect the appearance of many digital images. Such variations are due in magnetic resonance (MR) images to the inhomogeneity of the applied magnetic field, in microscopy images to nonuniform illumination and in underwater photography and long distance photography to the light absorption by the medium. For a review of a large number of methods applied to MRI images see [18]. In this paper we evaluate the performance of what we shall term the simplest possible gradient domain method to reduce the effect of nonuniform illumination while preserving fine image details. Since preserving fine image details is more or less equivalent to preserving the image gradient, the functional must have a forcing term of the type (1) with $\mathbf{V}=\nabla f$, where $f$ is the original image. To reduce the nonuniform illumination the simplest possible idea is to reduce the image variance, therefore adding in the variational method the term

$$
\int_{\Omega}(u-\bar{u})^{2} d x
$$

where $\bar{u}$ represents the mean value. We therefore consider the functional

$$
J(u)=\int_{\Omega}\|\nabla u-\nabla f\|^{2} d x+\lambda \int_{\Omega}(u-\bar{u})^{2} d x
$$

where $\lambda$ is a constant that controls the trade-off between both terms to be the simplest possible functional coping with the exigences of a Retinex like algorithm. Of course, after solving this variational problem, the variance being reduced, an affine stretch of the image dynamics can be effectuated, which is expected to enhance most image details.

To understand the behavior of the model, we consider a nonuniform illumination field (Figure 1) and apply the model with different values of $\lambda$. Note that the result with $\lambda=0$ coincides with the original image. When the value of $\lambda$ tends to infinity, the result becomes constant.

To better understand the model, Figure 2 shows the graph of a row of the image in Figure 1 and the result with $\lambda=0.001$ and $\lambda=0.01$. We will show that the variational model (2) boils down to a high pass filter. In the literature, one can find many such Wiener-like filters [15], [19], [3], [14]. The so called multiscale retinex methods [6] remove the low frequency illumination components while enhancing the high frequency reflectance components. They can also be seen as nonlinear high pass filters, and the same consideration applies to filters that try to model the human visual system [17], [5]. All of these mentioned models are far more complex than the proposed straightforward quadratic functional. Thus, it is fair to see if it compares to these more complex methods. While of course differences between results are apparent, on the whole we claim that the simple model is competitive. It can be tried in the online demo on any image, and compared to the online demo of ACE [5], which is considered one of the most effective contrast enhancement methods.

The paper is organized as follows. In Section 2 we present the variational model and we solve exactly in the Fourier domain the associated Euler-Lagrange equation of the model, which is a 

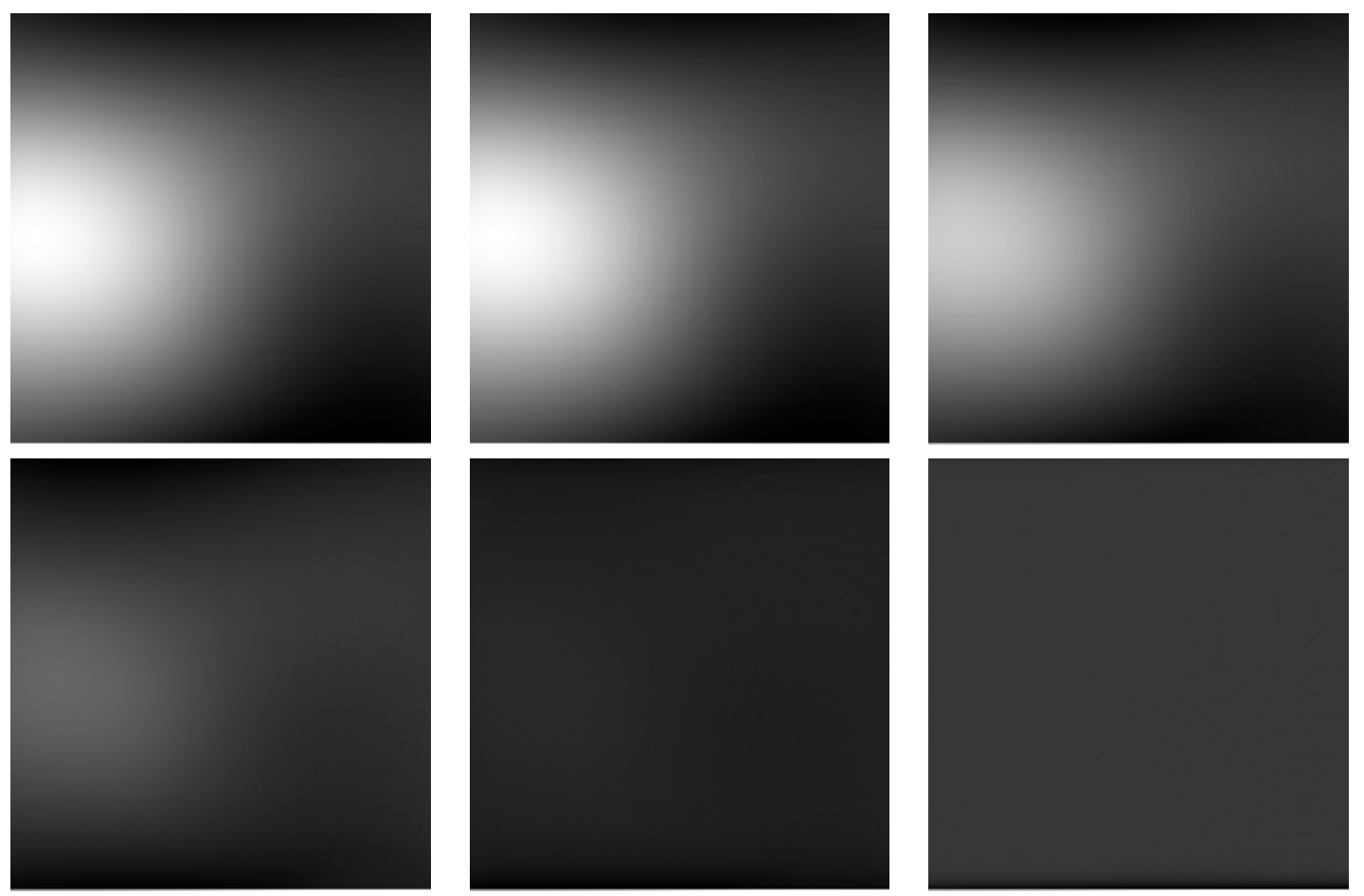

Figure 1: Inhomogeneous illumination. Result of the model with growing $\lambda=0,0.0001,0.001,0.01$, 0.1
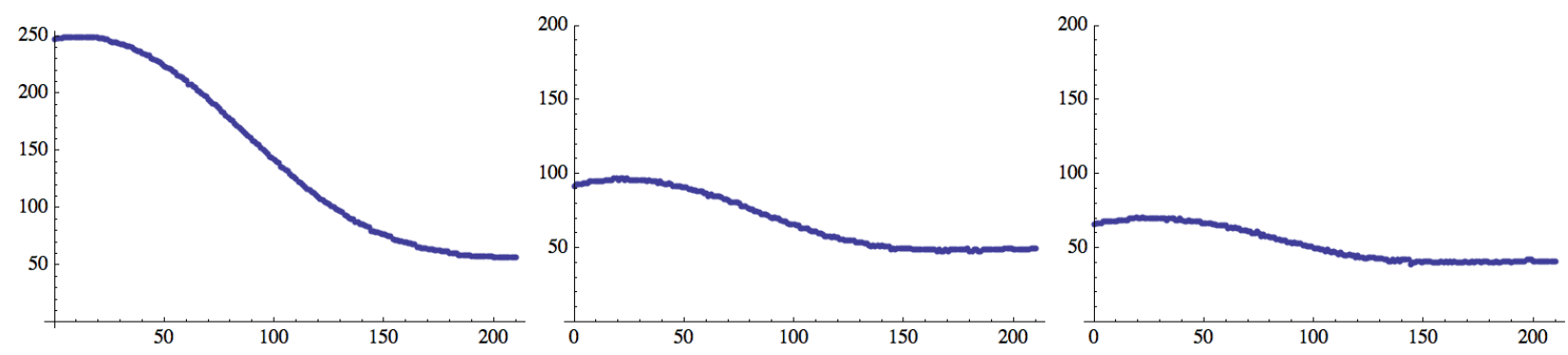

Figure 2: Left: graph of a row of the image in Figure 1. Middle: results with $\lambda=0.001$. Right: result with $\lambda=0.01$

screened Poisson equation. We prove that the spatial filter used to solve it is a specific high pass filter. A similar work is presented in [1], where the authors study, in the Fourier domain, the screened Poisson equation. In Section 3 we explain the algorithm to solve the screened Poisson equation using the fast Fourier transform. Section 4 presents some results and a discussion.

\section{The Model}

Given an image $f$, the goal is to find a function $u$ whose vector gradient is close to the vector gradient of $f$, but with reduced variance to compensate for illumination inhomogeneities. The objective function to minimize is

$$
J(u)=\int_{\Omega}\|\nabla u-\nabla f\|^{2} d x+\lambda \int_{\Omega}(u-\bar{u})^{2} d x,
$$

where $\lambda$ is a constant that controls the trade-off between the two terms. 
The Euler-Lagrange equation is nonlinear. Yet, it is immediately linearized by fixing that the mean value of $u$ coincides with the mean value of $f$. With this condition, the function $u$ that minimizes the functional $J$ satisfies the Euler-Lagrange equation

$$
\lambda(u-\bar{f})-\Delta u+\Delta f=0, \quad \text { over } \quad \Omega
$$

with homogeneous Neumann boundary condition

$$
\frac{\partial u}{\partial \mathbf{n}}=0, \quad \text { over } \quad \partial \Omega,
$$

where $\mathbf{n}$ is the normal vector to the boundary. Equation (4) is known as a screened Poisson equation.

\subsection{Independence of the Mean Value}

It remains to prove that imposing a mean value to the solution is irrelevant. Consider the problem

$$
\begin{cases}\lambda u-\Delta u=-\Delta f+\lambda K & \text { in } \Omega \\ \frac{\partial u}{\partial \mathbf{n}}=0 & \text { in } \partial \Omega\end{cases}
$$

where $K$ is a constant and $\lambda>0$. It is well known that problem (5) has a unique solution when $\lambda \neq 0$. Let $u_{1}$ and $u_{2}$ be two solutions of (5) with constants $K_{1}$ and $K_{2}$ respectively. Then it follows immediately from the uniqueness of the solution of (5) that

$$
u_{1}-u_{2}=K_{1}-K_{2} .
$$

Working (e.g.) with eight-bit digital images, the range of values of the image is the interval $[0,255]$. When solving problem (5) in the image context, the final solution must be stretched on this range, as mentioned above. This permits to benefit fully of the relative contrast gain attained by the method. Consider $u_{1}$ and $u_{2}$, solutions of (5) for two constants $K_{1}$ and $K_{2}$ respectively and let $\tilde{u_{1}}, \tilde{u_{2}}$ be the associated solutions in the range $[0,255]$. Setting $M_{i}=\max u_{i}$ and $m_{i}=\min u_{i}, i=1,2$ we have

$$
\tilde{u}_{i}=\frac{255}{M_{i}-m_{i}}\left(u_{i}-m_{i}\right), \quad i=1,2
$$

but $M_{1}=M_{2}+K_{1}-K_{2}$ and $m_{1}=m_{2}+K_{1}-K_{2}$, which implies $\tilde{u}_{1}=\tilde{u_{2}}$. We conclude that the final solution is independent of the constant $K$, so from now on we will set $K=0$.

\subsection{The Screened Poisson Equation in the Fourier Domain}

This section analyzes the solution of the screened Poisson equation

$$
\lambda u-\Delta u=-\Delta f
$$

as the result of a high pass filter of the function $f$, and more precisely as a "center-surround" filter, namely the subtraction from the image of the same image convolved with a local radial low pass filter. To this aim we analyze the equation in the Fourier domain. Given a function $f(x, y)$, its Fourier transform is defined by

$$
\widehat{f}\left(w_{x}, w_{y}\right)=\int_{\mathbb{R}^{2}} f(x, y) e^{-i x w_{x}} e^{-i y w_{y}} d x d y
$$


and the inverse Fourier transform by

$$
f(x, y)=\frac{1}{(2 \pi)^{2}} \int_{\mathbb{R}^{2}} \widehat{f}\left(w_{x}, w_{y}\right) e^{i x w_{x}} e^{i y w_{y}} d w_{x} d w_{y}
$$

Recall that

$$
\widehat{f_{x}}=i w_{x} \widehat{f}, \widehat{f_{y}}=i w_{y} \widehat{f}, \widehat{f_{x x}}=-w_{x}^{2} \widehat{f}, \widehat{f_{y y}}=-w_{y}^{2} \widehat{f} .
$$

Then transforming the screened equation gives

$$
\lambda \widehat{u}+\left(w_{x}^{2}+w_{y}^{2}\right) \widehat{u}=\left(w_{x}^{2}+w_{y}^{2}\right) \widehat{f},
$$

and solving for $\widehat{u}$ yields

$$
\widehat{u}=\frac{w_{x}^{2}+w_{y}^{2}}{\lambda+w_{x}^{2}+w_{y}^{2}} \widehat{f} .
$$

The frequency filter acts as a high pass filter when $\lambda$ increases as illustrated in Figure 3.

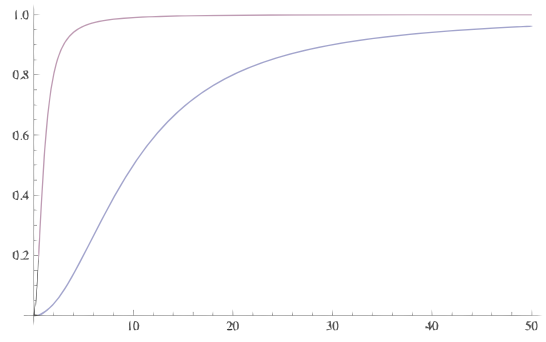

Figure 3: The frequency domain filter for $\lambda=0.1$ (in red) and $\lambda=50$ (in blue).

\subsection{The Solution in the Spatial Domain}

Starting from Equation (6) the frequency filter can be decomposed as

$$
\frac{w_{x}^{2}+w_{y}^{2}}{\lambda+w_{x}^{2}+w_{y}^{2}}=1-\frac{\lambda}{\lambda+w_{x}^{2}+w_{y}^{2}} .
$$

Computing the inverse Fourier transform of this filter indicates that the solution of the screened Poisson equation is obtained by convolving the image $f$ with a spatial domain filter. More precisely, the inverse Fourier transform of the first term in (7) is a Dirac delta function $\delta(x, y)$. The second term is radially symmetric and interpretable using the Hankel transform [2]. Denoting by $\mathcal{H}\{f\}$ the Hankel transform of $f(r)$, we have

$$
\mathcal{H}\{f\}(\rho)=\int_{0}^{\infty} r f(r) J_{0}(r \rho) d r
$$

and the inverse Hankel transform is

$$
f(r)=\int_{0}^{\infty} \rho \mathcal{H}\{f\}(\rho) J_{0}(r \rho) d \rho,
$$

where $J_{0}$ is the zeroth order Bessel function of first kind.

If $f(x, y)$ is radially symmetric, that is, if $f$ only depends on $r=\sqrt{x^{2}+y^{2}}$, then the 2D Fourier transform satisfies

$$
\widehat{f}\left(w_{x}, w_{y}\right)=2 \pi \mathcal{H}\{f\}(\rho), \quad \rho=\sqrt{w_{x}^{2}+w_{y}^{2}} .
$$


The inverse Fourier transform of a radially symmetric function satisfies

$$
f(x, y)=\frac{1}{2 \pi} \mathcal{H}^{-1}\{\widehat{f}\}(r), \quad r=\sqrt{x^{2}+y^{2}} .
$$

It is known that

$$
\mathcal{H}^{-1}\left\{\frac{1}{a^{2}+\rho^{2}}\right\}=K_{0}(\text { ar })
$$

where $K_{0}$ is the zeroth order modified Bessel function of second kind. Then the inverse Fourier transform of the frequency filter (7) is

$$
\delta(x, y)-\frac{1}{2 \pi} K_{0}\left(\sqrt{\lambda\left(x^{2}+y^{2}\right)}\right) .
$$

Convolving the filter (8) with $f$ yields the solution of the screened Poisson equation

$$
u(x, y)=f(x, y)-\left(\frac{1}{2 \pi} K_{0}\left(\sqrt{\lambda\left(x^{2}+y^{2}\right)}\right) * f(x, y)\right) .
$$

Thus, the solution is the difference between the data function and a blurred version of it with the $K_{0}$ filter. This filter acts as a center-surround filter, as illustrated in Figure 4. Center-surround filters of this kind model the human perception of colors [7], [1].

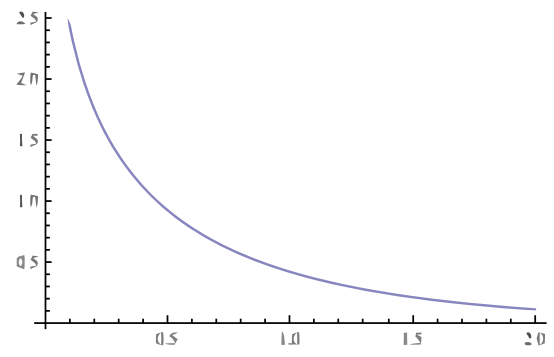

Figure 4: The zeroth order modified Bessel function of second kind $K_{0}(r)$

\section{Algorithm}

We want to solve the problem

$$
\begin{cases}\lambda u-\Delta u=-\Delta f & \text { in } \Omega \\ \frac{\partial u}{\partial \mathbf{n}}=0 & \text { in } \partial \Omega\end{cases}
$$

where $\lambda>0$. We can solve the screened Poisson equation (10) using the discrete Fourier transform. Like in [15], the Neumann boundary condition is implicitly imposed by extending the original image symmetrically across its sides, so that the extended image, which is four times bigger, becomes symmetric and periodic. The discrete Fourier transform (DFT) permits to compute directly the Fourier coefficients of a band-limited and $(J, L)$-periodic function $u$ from its samples $u_{j l}$ on a $J \times L$ grid. The output of the algorithm is an image which is the result of applying the screened Poisson equation to each color channel separately, followed by a simplest color balance [11] with a $0.2 \%$ of saturation. Then the screened Poisson becomes in the Fourier domain

$$
\left(\lambda+\left(\frac{2 \pi m}{J}\right)^{2}+\left(\frac{2 \pi n}{L}\right)^{2}\right) \widehat{u}_{m n}=\left(\left(\frac{2 \pi m}{J}\right)^{2}+\left(\frac{2 \pi n}{L}\right)^{2}\right) \widehat{f}_{m n} .
$$


The solution in the Fourier domain is given by

$$
\widehat{u}_{m n}=\frac{\left(\left(\frac{2 \pi m}{J}\right)^{2}+\left(\frac{2 \pi n}{L}\right)^{2}\right)}{\left(\lambda+\left(\frac{2 \pi m}{J}\right)^{2}+\left(\frac{2 \pi n}{L}\right)^{2}\right)} \widehat{f}_{m n} .
$$

Applying the discrete inverse Fourier transform yields the solution $u$.

In eight bit digital images the range is $[0,255]$. The best results are obtained when a certain small percentage of pixels are saturated at 255 and 0 , as shown in [11]. The proposed model is applied to the result of a simplest color balance with $0.2 \%$ saturation, and the final result is obtained by applying to the solution a simplest color balance again. The proposed algorithm is described as follows

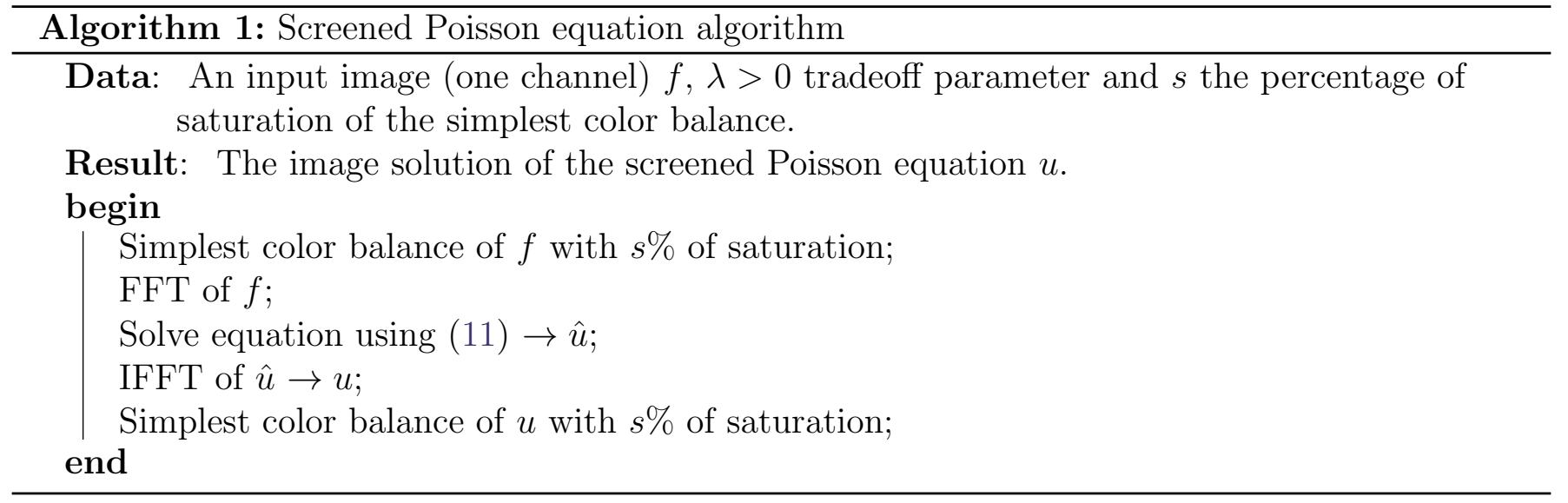

Remark that this algorithm is applied on monochrome images after symmetrization, as indicated above. For RGB images it applies on each color channel independently. For the Fourier transform we use the library $\mathrm{fftw}^{2}$. In fact with the FFTW library the symmetrization is implicit and performed directly as a cosine transform. The complexity of the algorithm is $O(n \log n)$. The CPU time on an intel core i7, 2.7 Ghz and 4 Gb of RAM is 0.63 seconds for a $700 \times 528$ image.

\section{Results}

In this section we present some applications of the model. These include non uniform illumination correction, contrast enhancement, and edge detection.

\subsection{Non Uniform Illumination Correction}

Figure 5 shows an example of a text image with nonuniform illumination. In medical imaging inhomogeneous scene illumination is frequent and can cause dark regions with low contrast. This results in a smooth intensity variation across the image which can be compensated. For example in ophthalmology the use of advanced fundus cameras has become a standard imaging modality to diagnose diseases such as glaucoma, diabetic retinopathy etc. A better contrast of the relevant objects on the retina such as the blood vessels or the optic disk can be obtained by restoring the uniformity of the retina illumination. The classic methods estimate the illumination or the bias field, using the commonly used terminology, and subtract it from the original image to obtain the final result [9], [8].

Figure 6 shows an example of a retina image and the result after applying our model. We compare the results with the above mentioned classic method [8]. Clearly the blood vessels are more visible

\footnotetext{
${ }^{2}$ M. Frigo and S.G. Johnson,FFTW package, http://fftw.org
} 

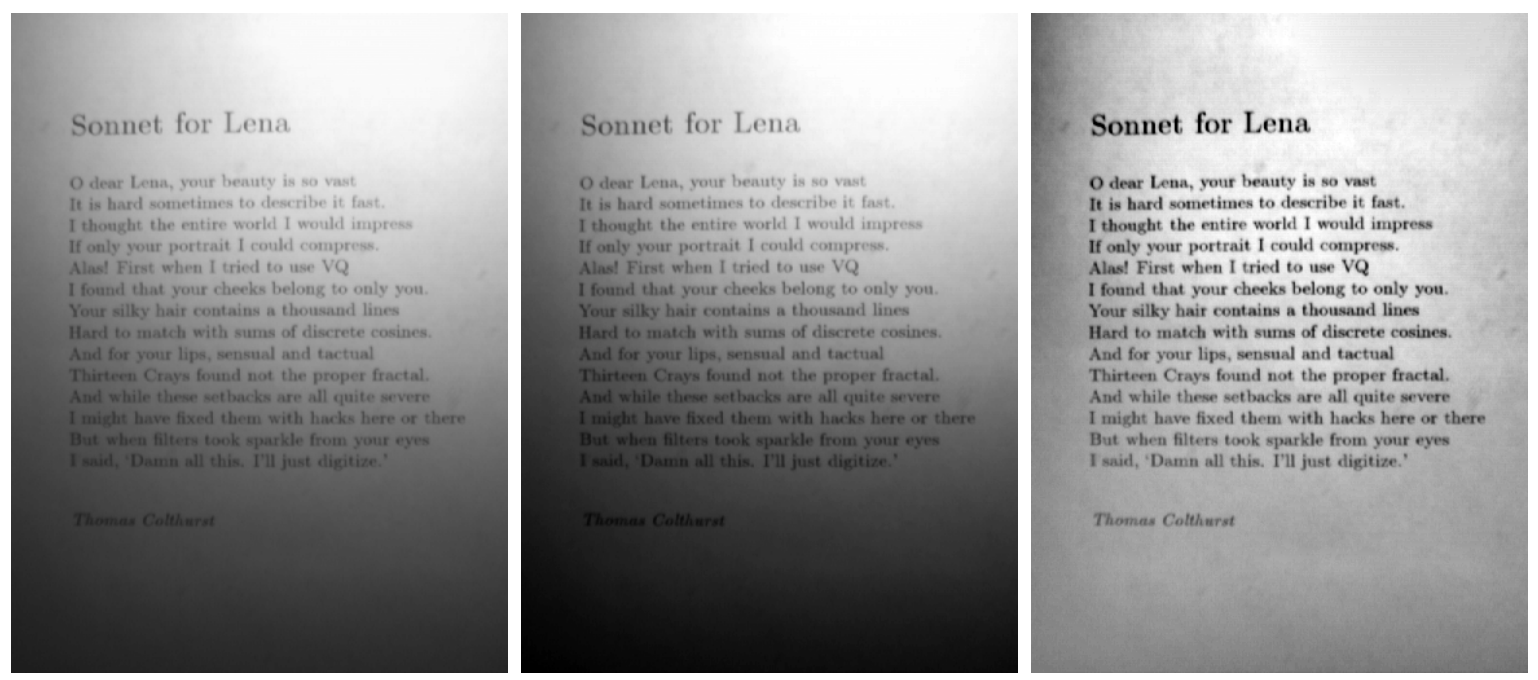

Figure 5: Left: original Image. Middle: result of simplest color balance with $0.2 \%$ of saturation. Right: result with our model with $\lambda=0.0005$

in dark regions and the result can be used as a preprocessing step in the blood vessel segmentation. The image and the result of the method in [8] come from the public fundus images database DRIIL (Digital Retinal Images for ILlumination correction ${ }^{3}$ ).
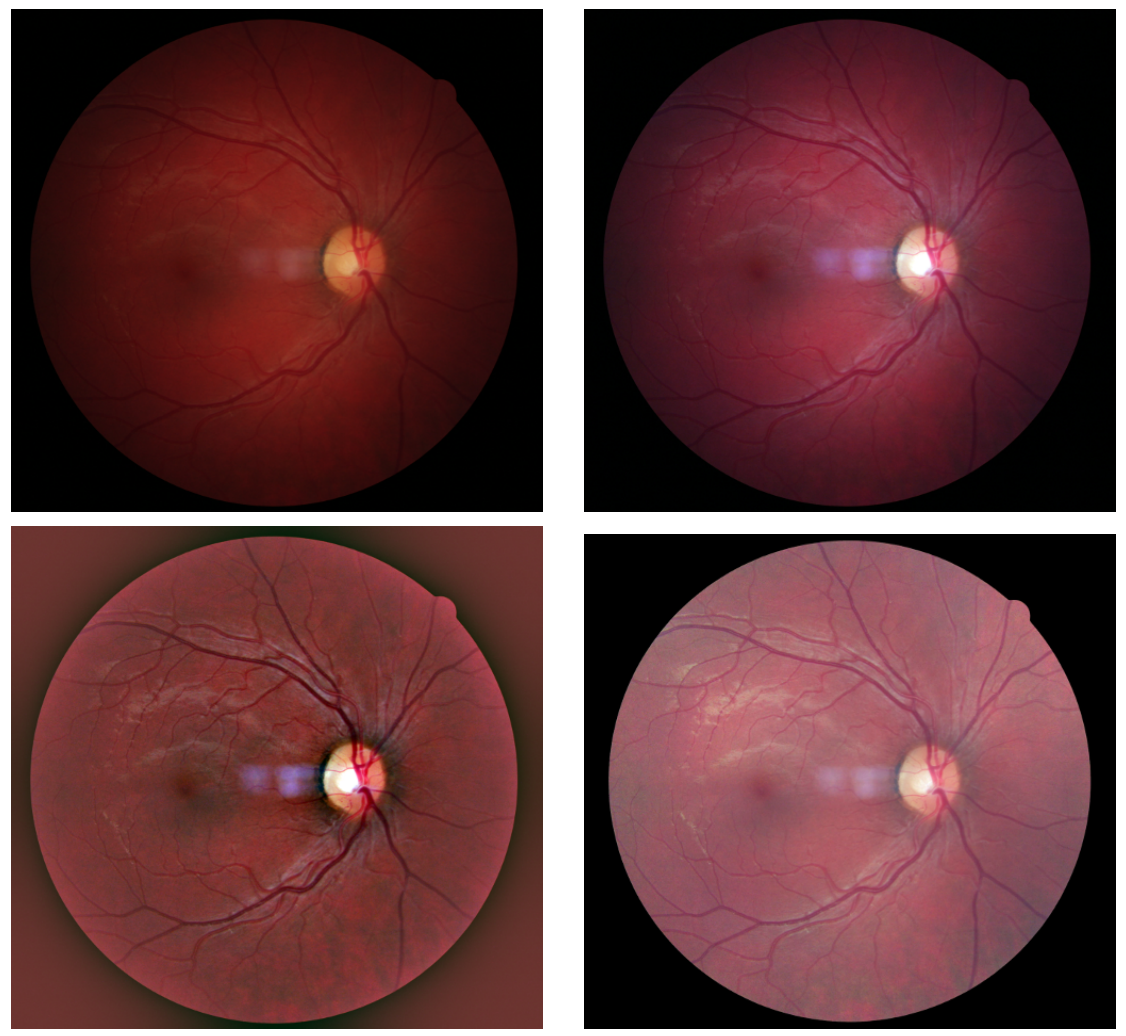

Figure 6: Top left: original Image. Top right: result of simplest color balance with $0.2 \%$ of saturation. Bottom left: result with our model with $\lambda=0.0005$. Bottom right: result of the model. [8]

\footnotetext{
${ }^{3}$ http://projects.ubmi.feec.vutbr.cz/ophthalmo
} 
Another example for potential applications is given by magnetic resonance images (MRI), where the intensity inhomogeneity is due for example to radio frequency inhomogeneities or to variations in the static magnetic field. Vovk et al. [18] propose a classification of the correction methods into prospective methods, that attempt to improve the image acquisition process, and the retrospective methods that work with the information of the acquired image. The retrospective methods can be classified into filtering, surface fitting, segmentation and histogram based. The filtering methods assume that the nonuniform illumination is a low frequency artifact that must be removed. This is achieved by subtracting from the image its low-pass filtered version. To this group belong the homomorphic filtering and the unsharp masking. Our method belongs to this group, as explained in Section 2.3. Note that when $\lambda$ increases, the support of this filter becomes smaller.

Figures 7 and 8 show the result of our model on MRI images with intensity inhomogeneities. Clearly the dark regions become more visible and image details are preserved.
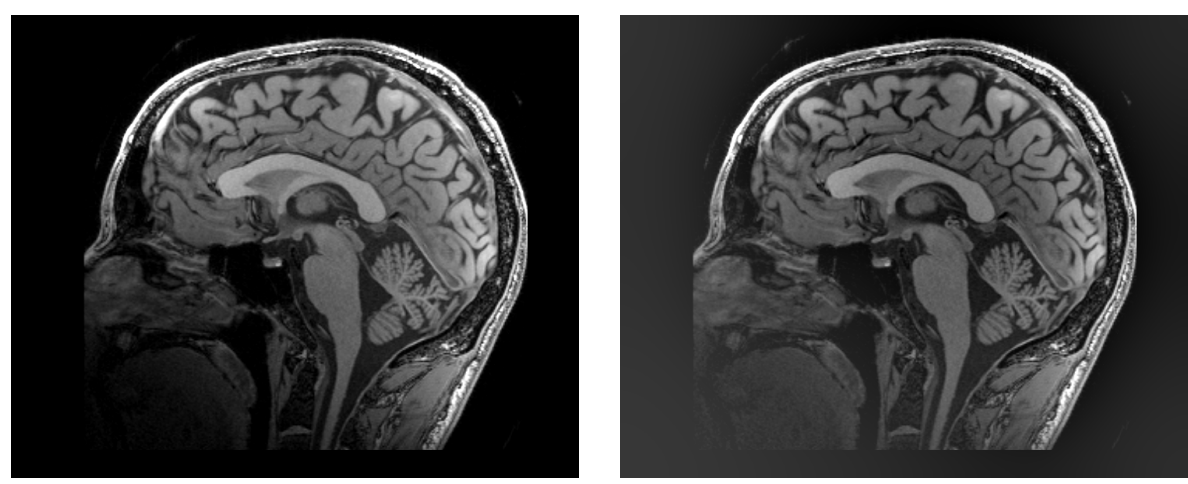

Figure 7: Left: original image. Right: result with $\lambda=0.0005$.
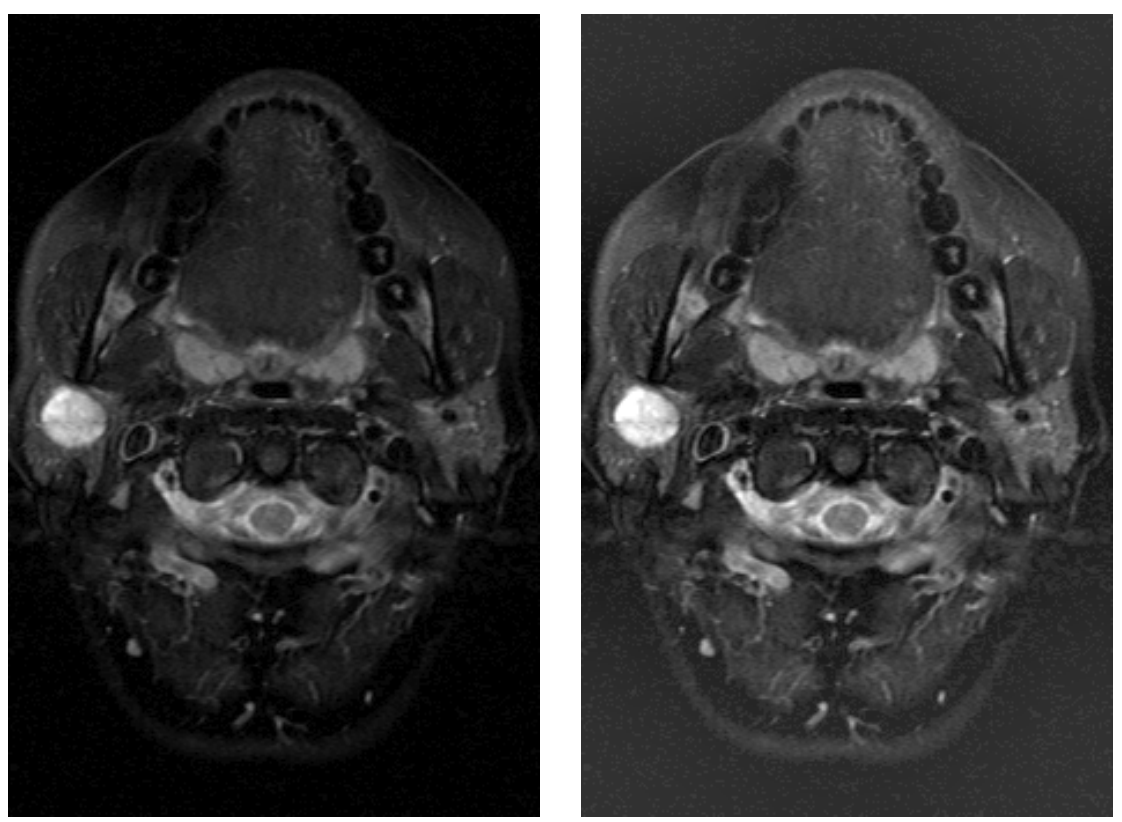

Figure 8: Left: original image. Right: result with $\lambda=0.0005$. 


\subsection{Contrast Enhancement Results}

The recently proposed contrast enhancement methods give increasingly good results [11], [15], [13], [6], [17], etc. In this section we compare our result with the state of the art automatic color enhancement (ACE) [17] and with the equally classic multiscale retinex with color restoration (MSRCR) [6]. ACE results were obtained from the online fast implementation of this algorithm [5] and we have implemented our own (MSRCR).

Figure 9 presents a low contrast image and the corresponding results of ACE, MSRCR and our model with parameter $\lambda=0.0001$. Observe that the high frequencies (for example in the letters on the fire-fighter helmet) are better preserved with our model. Another example of contrast enhancement
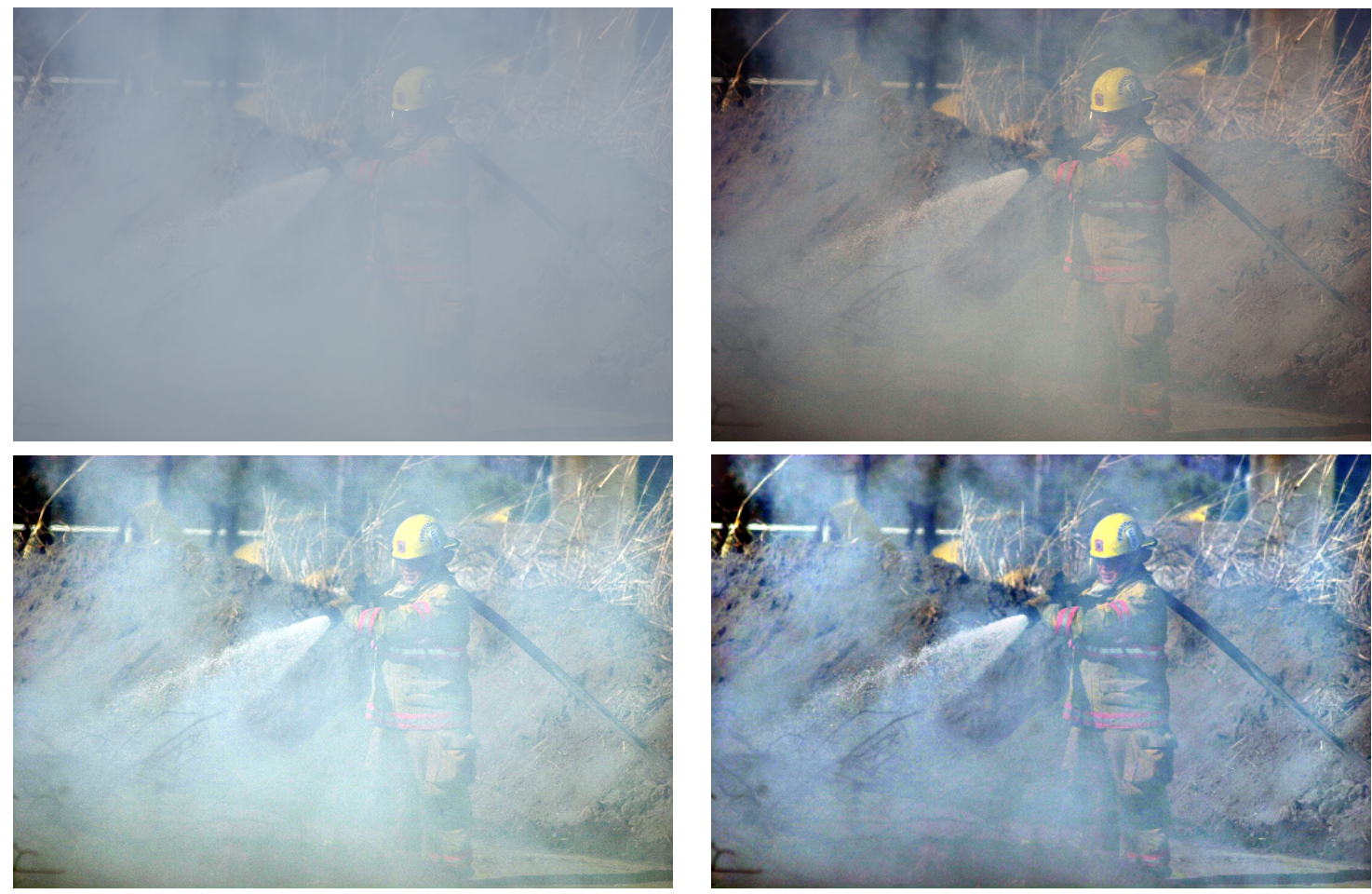

Figure 9: Top left: original Image. Top right: result of ACE with $\alpha=8$. Bottom left: result with MSRCR. Bottom right: our model with $\lambda=0.0001$.

is shown in Figure 10. Observe that the best result in this case is with the ACE method as the MSR method gives a very saturated image.

\subsection{Tuning $\lambda$}

In unsharp masking the original image is enhanced by subtracting a smooth version of the original image. Figure 11 shows the results with increasing values of $\lambda$. For a small value of $\lambda$ the gradient term is more important and the result is similar to a simplest color balance. As $\lambda$ increases, the histogram is more and more concentrated around the mean value but the gradients are preserved. The final simplest color balance stretches the dynamic range and produces a better contrasted image.

Figure 12 shows an example with a retina image when $\lambda$ tends to infinity. The result shows that when $\lambda$ is too large the models acts as an edge detector. 

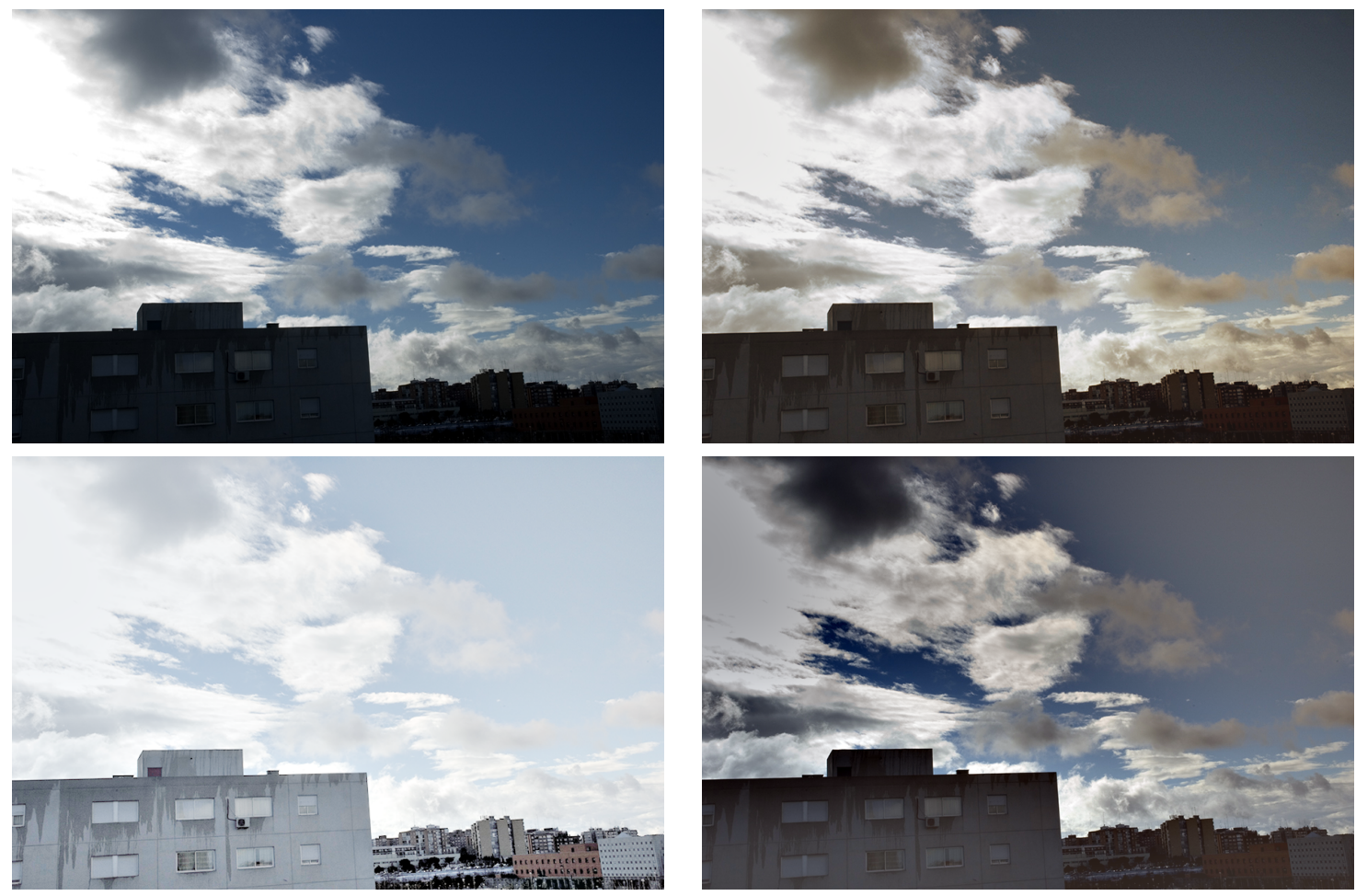

Figure 10: Top left: original Image. Top right: result of ACE. Bottom left: result with MSRCR. Bottom right: our model with $\lambda=0.0001$.
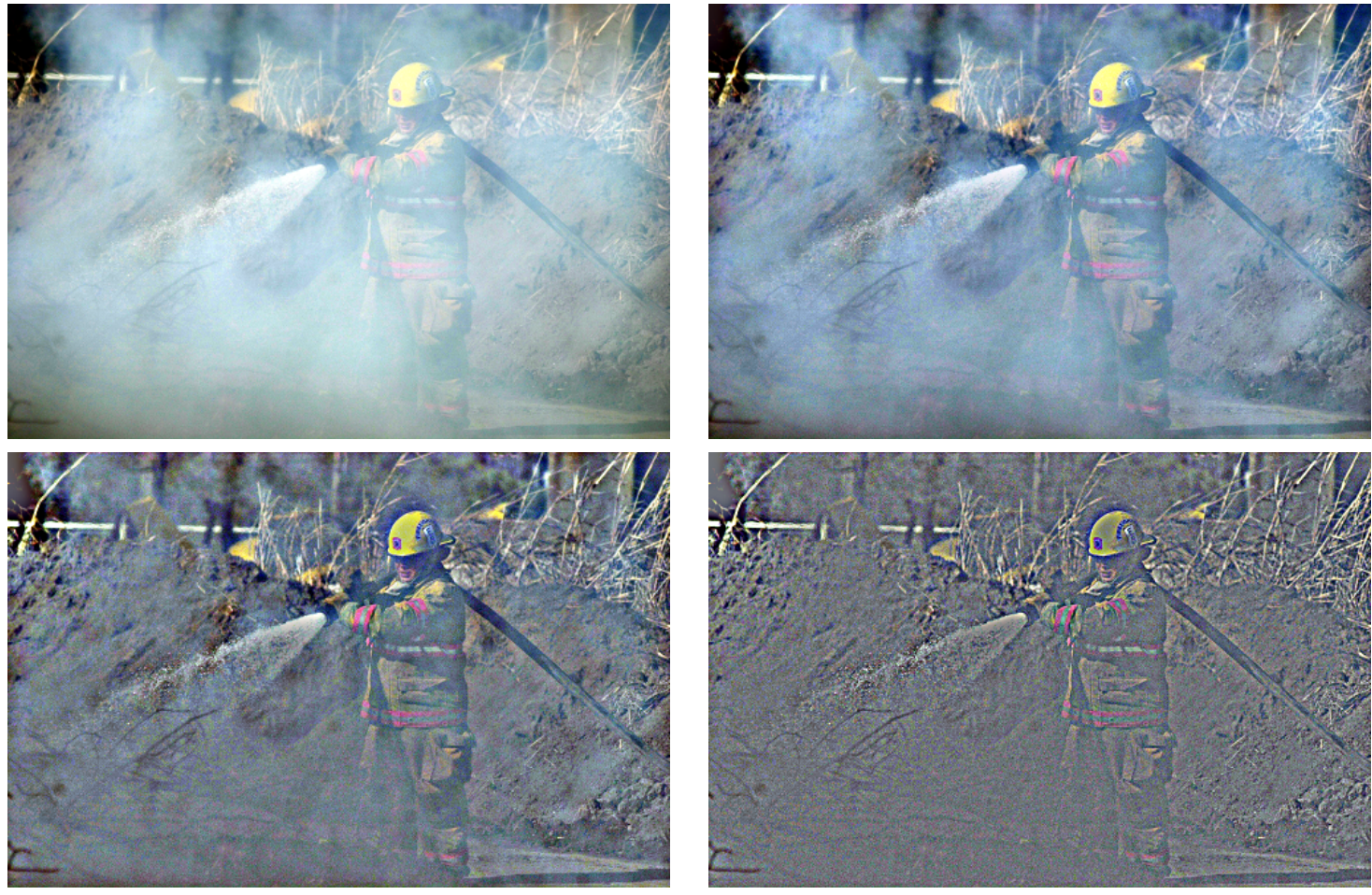

Figure 11: Top left : result with $\lambda=0.0001$. Top right: result with $\lambda=0.0005$. Bottom left: result with $\lambda=0.001$. Bottom right: result with $\lambda=0.005$. 

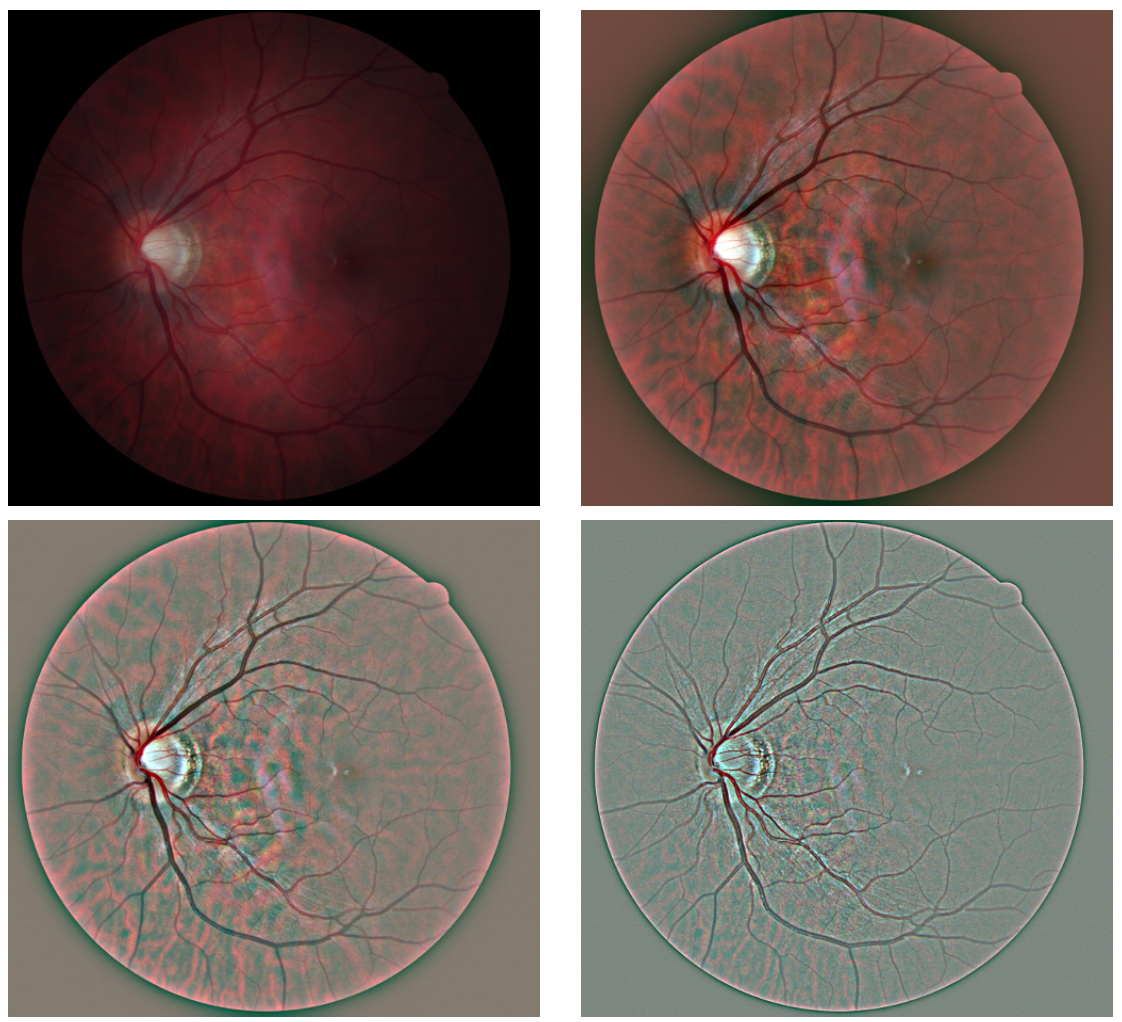

Figure 12: Top left : original image. Top right: result with $\lambda=0.0001$. Bottom left: result with $\lambda=0.01$. Bottom right: result with $\lambda=0.1$.

\section{Acknowledgments}

The authors were supported by the Ministerio de Ciencia e Innovación under Grant TIN2011-27539, by MISS project of Centre National d'Etudes Spatiales, the Office of Naval Research under Grant N00014-97-1-0839 and by the European Research Council, advanced grant "Twelve labours".

\section{Image Credits}

Photography courtesy Fisher, R. B., Perkins, S., Walker, A., Wolfart, E. .

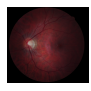

C. Public images database DRIIL ${ }^{5}$.

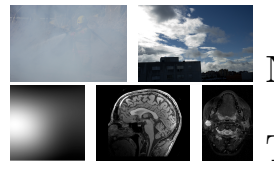

$\mathrm{NASA}^{6}$.

The authors CC-BY.

\section{References}

[1] P. Bhat, B. Curless, M.Cohen, and C. Lawrence, Fourier analysis of the $2 D$ screened Poisson equation for gradient domain problems, in Proceedings of the 10th European Con-

\footnotetext{
${ }^{4}$ HIPR: Hypermedia Image Processing Reference http://homepages.inf.ed.ac.uk/rbf/HIPR2/library.htm

${ }^{5}$ Digital Retinal Images for ILlumination correction http://projects.ubmi.feec.vutbr.cz/ophthalmo

${ }^{6}$ http://dragon.larc.nasa.gov/retinex/
} 
ference on Computer Vision, part II, 2008, pp. 114-128. http://dx.doi.org/10.1007/ 978-3-540-88688-4_9.

[2] R. Bracewell, The Fourier Transform and Its Applications, Mcgraw-Hill, 3rd ed., 1999. ISBN-13: 978-0073039381.

[3] R. Fattal, D. Lischinski, And M. Werman, Gradient domain high dynamic range compression, in Proceedings of the 29th Annual Conference on Computer Graphics and Interactive Techniques, SIGGRAPH '02, ACM, 2002, pp. 249-256. http://doi.acm.org/10.1145/ 566570.566573.

[4] G.D. Finlayson, S.D. Hordley, And M.S. Drew, Removing shadows from images, in Computer Vision ECCV 2002, vol. 2353 of Lecture Notes in Computer Science, Springer Berlin Heidelberg, 2002, pp. 823-836. http://dx.doi.org/10.1007/3-540-47979-1_55.

[5] P. Getreuer, Automatic Color Enhancement (ACE) and its Fast Implementation, Image Processing On Line, 2012 (2012), pp. 266-277. http://dx.doi.org/10.5201/ipol.2012.g-ace.

[6] D.J. Jobson, Z. Rahman, And G.A. Woodell, A multiscale retinex for bridging the gap between color images and the human observation of scenes, IEEE Transactions on Image Processing, 6 (1997), pp. 965-976. http://dx.doi.org/10.1109/83.597272.

[7] D. J. Jobson, Z. Rahman, And G. A. Woodell, Properties and performance of a center/surround retinex, IEEE Transactions on Image Processing, 6 (1997), pp. 451-462. http: //dx.doi.org/10.1109/83.557356.

[8] R. Kolar, J. Odstrcilik, J. Jan, And V. Harabis, Illumination correction and contrast equalization in colour fundus images, in Proceedings of 19th European Signal Processing Conference (EUSIPCO) Barcelona, Spain, 2011, pp. 298-302.

[9] L. Kubecka, J. Jan, And R. KolaR, Retrospective illumination correction of retinal images, International Journal of Biomedical Imaging, vol. 2010, 10 pages, (2010). http://dx.doi.org/ $10.1155 / 2010 / 780262$

[10] E.H. LAND, The Retinex theory of color vision, Scientific American, 236 (1977), pp. 108-128.

[11] N. Limare, J.L. Lisani, J.M. Morel, A.B. Petro, and C. Sbert, Simplest Color Balance, Image Processing On Line, (2011). http://dx.doi.org/10.5201/ipol.2011.1lmps-scb.

[12] N. Limare, A.B. Petro, C. Sbert, And J.M. Morel, Retinex Poisson Equation: a Model for Color Perception, Image Processing On Line, (2011). http://dx.doi.org/10.5201/ipol. 2011.lmps_rpe.

[13] J.L. Lisani, A.B. Petro, and C. Sbert, Color and Contrast Enhancement by Controlled Piecewise Affine Histogram Equalization, Image Processing On Line, (2012), pp. 243-265. http: //dx.doi.org/10.5201/ipol.2012.1ps-pae.

[14] J.M. Morel, A.B. Petro, and C. Sbert, A PDE formalization of Retinex theory, IEEE Transactions on Image Processing, 19 (2010), pp. 2825-2837. http://dx.doi.org/10.1109/ TIP. 2010.2049239.

[15] J.M. Morel, A.B. Petro, And C. Sbert, Fourier implementation of Poisson image editing, Pattern Recognition Letters, 33 (2012), pp. 342 - 348. http://dx.doi.org/10.1016/j . patrec.2011.10.010. 
[16] P. PÉrez, M. Gangnet, And A. Blake, Poisson image editing, in Proceedings of the 30th Annual Conference on Computer Graphics and Interactive Techniques, SIGGRAPH '03, ACM, 2003, pp. 313-318. http://doi.acm.org/10.1145/1201775.882269.

[17] A. Rizzi, C. Gatta, And D. Marini, A new algorithm for unsupervised global and local color correction, Pattern Recognition Letters, 24 (2003), pp. 1663 - 1677. Colour Image Processing and Analysis. First European Conference on Colour in Graphics, Imaging, and Vision (CGIV 2002).

[18] U. Vovk, F. Pernus, And B. Likar, A review of methods for correction of intensity inhomogeneity in MRI, IEEE Transactions on Medical Imaging, 26 (2007), pp. 405-421. http://dx.doi.org/10.1109/TMI.2006.891486.

[19] M. Wenye, J.M. Morel, S. Osher, And A. Chien, An L1-based variational model for retinex theory and its application to medical images, in Proceedings of IEEE Conference on Computer Vision and Pattern Recognition (CVPR), June 2011, pp. 153-160. http://dx. doi. org/10.1109/CVPR.2011.5995422. 